\title{
Genome-scale identification of MLO domain-containing genes in soybean (Glycine $\max$ L. Merr.)
}

\author{
Qi Shen ${ }^{1,2 \dagger}$, Jinming Zhao ${ }^{1 \dagger}$, Caifu Du ${ }^{2}$, Yang Xiang ${ }^{1,2 *}$, \\ Jinxuan $\mathrm{Cao}^{3}$ and Xinrong Qin ${ }^{2}$ \\ ${ }^{1}$ National Key Laboratory of Crop Genetics and Germplasm Enhancement, Nanjing Agricultural University, \\ Nanjing 210095, P.R.China \\ ${ }^{2}$ Guizhou Rapeseed Institute, Guizhou Academy of Agricultural Sciences, Guiyang 550008, P.R.China \\ ${ }^{3}$ College of Life Science and Biotechnology, Ningbo University, Ningbo 315211, P.R.China.
}

(Received 9 June 2011, accepted 27 February 2012)

In plants, powdery-mildew-resistance locus o (Mlo) genes encode proteins that are calmodulin-binding proteins involved in a variety of cellular processes. However, systematic characterization of this gene family in soybean (Glycine max L. Merr.) has not been yet reported. In this study, we identified MLO domaincontained members in soybean and examined their expression under phytohormone treatment and abiotic stress conditions. A total of 20 soybean Mlo genes were identified (GmMlo1-20), which are distributed on 13 chromosomes, and display diverse exon-intron structures. Phylogenetic analysis indicated that the Mlo family can be classified into four subfamilies. Sequence comparison was used to reveal the conserved calmodulin-binding domain (CaMBD) in GmMLO proteins. The expression of $\mathrm{GmMlo}$ genes was influenced by various phytohormone treatments and abiotic stresses, suggesting that these Mlo genes have various roles in the response of soybean to environmental stimuli. Promoter sequence analysis revealed an overabundance of stress and/or phytohormone-related cis-elements in $\mathrm{GmMlo}$ genes. These data provide important clues for elucidating the functions of genes of the Mlo gene family.

Key words: soybean; MLO domain; gene family; abiotic stress; phytohormone

\section{INTRODUCTION}

In plant cells, the calcium ion $\left(\mathrm{Ca}^{2+}\right)$ is a ubiquitous intracellular second messenger involved in numerous signaling pathways. It also affects diverse cellular and developmental processes, including modulation of apoptosis, stress responses, organogenesis and transcriptional activity (Yang and Poovaiah, 2003). Calcium signaling is decoded and transduced by an array of $\mathrm{Ca}^{2+}$-binding proteins, giving rise to a cascade of downstream calciumdependent events (Rudd and Franklin-Tong, 2001; Sanders et al., 2002). Some genes have been identified that are involved in the early stage of calcium signaling transduction; for example, calmodulin $(C a M)$, pathogen-induced CaM-binding protein $(P I C B P)$, calcineurin B-like $(C B L)$, powdery mildew resistance locus o (Mlo), Arabidopsis thaliana stress responsive genes (AtSR), calciumdependent protein kinases $(C D P K s)$ and CaM-binding protein $60 \mathrm{~g}$ (CBP60g) (Büschges et al., 1997; Zielinski,

\footnotetext{
Edited by Jaroslav Dolezel

* Corresponding author. E-mail: xiangyangcell@yahoo.com

$\dagger$ These authors contributed equally to this work
}

1998; Reddy, 2001; Hrabak et al., 2003; Du et al., 2009; Wang et al., 2009).

Mlo genes are a particular class of plant-specific CaMbinding protein genes (Wolter et al., 1993; Kim et al., 2002a, 2002b). The Mlo gene was first isolated in barley (Büschges et al., 1997) and, since then, genes encoding MLOs have been identified in several plant species, including 15 Mlos in Arabidopsis thaliana, seven in wheat and 12 in rice (Devoto et al., 2003; Liu and Zhu, 2008; Konishi et al., 2010). Accumulating data indicate that MLO proteins have a characteristic structure comprising seven transmembrane (TM) domains and a Cterminal CaM-binding domain (CaMBD) (Büschges et al., 1997; Devoto et al., 1999; Kim et al., 2002a, 2002b). Binding of CaM to MLO mediates a $\mathrm{Ca}^{2+}$-dependent pathogen defence reaction (Kim et al., 2002a, 2002b). Thus, the $M l o$ gene is known as an early $\mathrm{Ca}^{2+}$-responsive gene.

Recently, Mlos have attracted increasing attention because of their association with the stress responses in plants. In barley (Hordeum vulgare), homozygous recessive mutant alleles of the Mlo gene can confer durable and broad-spectrum pathogen resistance to the biotrophic 
powdery mildew fungus (Jørgensen, 1992; Piffanelli et al., 2002, 2004). Loss of Arabidopsis MLO2 plant produced resistance against multiple powdery mildew species (Consonni et al., 2006, 2010). The same defense mechanism was found in tomato MLO1 (Bai et al., 2008). Another main role of $M l o$ in plants is in the regulation of cell death; for example, mutant mlo plants exhibit spontaneous mesophyll cell death associated with partly accelerated leaf senescence (Wolter et al., 1993; Piffanelli et al., 2002; Stein and Somerville, 2002; Consonni et al., 2010). An increasing number reports suggest that Mlos also have additional functions in response to abiotic stimuli. For example, there are some evidences to suggest that $M l o$ transcript abundance increased in response to wounding, paraquat treatment, a wheat powdery mildew-derived carbohydrate elicitor, salt stress and mannitol treatment (Piffanelli et al., 2002; Feechan et al., 2008; Konishi et al., 2010). However, despite the apparent association of $M L O s$ with plant abiotic stresses, their roles in plants remain elusive.

Soybean (Glycine $\max$ L. Merr.) is one of the most economically oil crops worldwide. Based on a comparison with MLOs in other plant species, those in soybean should also be encoded by the same multi-gene family. However, Mlo genes have not yet been identified in soybean. Sequencing of the soybean genome provides an opportunity to identify previously uncharacterized genes. In this study, a bioinformatics approach was used to identify all members of the Mlo gene family in soybean. Their genomic organization, $\mathrm{Ca}^{2+}$-binding motif location, phylogenesis, cis-elements in promoter regions and the expression profiles under various abiotic and hormone stresses were analyzed in detail. The results presented here provide important information for future function studies of Mlo genes.

\section{MATERIALS AND METHODS}

Identification of Mlo members in soybean The HMMER (version 2.3.2) program was used to search all MLO domain-containing protein genes in the soybean genome databases (http://www.phytozome.net/soybean.php, phytozome, Release v4.0) with the HMM (Hidden Markov Model) profile of the MLO domain (PF03094) (http:// pfam.sanger.ac.uk/Software/Pfam; Finn et al., 2006) and with an E-vlaue $<1.0 \mathrm{e}-200$ and more than 450 amino acid residues as the cutoff. The predicted MLO sequences were then compared against the conserved MLO domain in the Pfam and SMART (http://smart.embl-heidelberg. de; Letunic et al., 2004) databases.

Database search and sequence analysis Expressed sequence tags (ESTs) were subjected to a BLAST search against the identified $M l o$ sequences to determine genuine EST hits. The exons-introns structures in the differ- ent genes were determined by comparing of the cDNAs with their corresponding genomic DNA sequences. Open reading frame $(\mathrm{ORF})$ analyses were performed with the ORF Finder (http://www.ncbi.nlm.nih.gov/gorf/gorf.html). The exons and introns were determined by using the Gene Structure Display Server (GSDS) tool (http:// gsds.cbi.pku.edu.cn/). TM segments were predicted by using the TMHMM2 tool (http://www.cbs.dtu.dk/services/ TMHMM/; Krogh et al., 2001). Motifs were identified statistically using online MEME (http://meme.sdsc.edu). CaM-binding motif analysis was identified according to Kim et al. (2002b). The chromosomal positions of GmMlo genes were also retrieved from phytozome soybean genome databases (http://www.phytozome.net/soybean.php, phytozome). Ten proteins-encoding genes from regions flanking of each GmMlo gene were retrieved from phytozome soybean genome databases to identify segmental replication events of $\mathrm{GmMlos}$.

Protein sequence alignments and phylogenetic analysis Multiple alignments of protein sequences were performed using Clustal W (Accelrys Inc., USA). A phylogenetic tree was constructed using neighbour-joining (NJ) algorithms. Bootstrapping was performed 1000 times to obtain support values for each branch.

cis-elements identification To analyze putative cisacting regulatory DNA elements (i.e. cis-elements) in the promoters of GmMlo genes, online PLACE 26.0 (http:// www.dna.affrc.go.jp/PLACE/index.html; Lescot et al., 2002) was used to analyze a 2,000-bp sequence upstream of the full-length cDNAs or predicted CDS extracted from the genomic sequences.

Plant materials, abiotic and phyhormone treatments The plant materials used in this study included the soybean cultivars 'Williams'. Plants were grown in a controlled environment chamber $\left(200 \mu \mathrm{mol}\right.$ photons $\mathrm{m}^{-2} \mathrm{~s}^{-1}$, $14 \mathrm{~h}$ light/10 $\mathrm{h}$ dark per day at $22 \pm 2^{\circ} \mathrm{C}$ ).

In the abiotic treatment, 14-day-old seedlings were treated at either $40^{\circ} \mathrm{C}$ or $4^{\circ} \mathrm{C}$, or with either $5 \%$ polyethylene glycol (PEG) or $200 \mathrm{mM} \mathrm{NaCl}$. In the $40^{\circ} \mathrm{C}$ treatment, the leaves were freeze-stored $1 \mathrm{~h}$ after the heat shock treatment. In the $4^{\circ} \mathrm{C}$ treatment, the leaves were collected $6 \mathrm{~h}$ after treatment. For the PEG and $\mathrm{NaCl}$ treatments, the leaves were then frozen in liquid nitrogen after $24 \mathrm{~h}$ of treatment. Untreated seedlings were used as controls.

To obtain phytohormones-treated leaf tissues, 14-dayold seedling leaves were sprayed with either $10 \mathrm{mM}$ abscisic acid (ABA), $1 \mathrm{mM}$ 6-benzyladenine (6-BA), $10 \mathrm{mM}$ gibberellic acid $\left(\mathrm{GA}_{3}\right)$ or $1 \mathrm{mM}$ indole acetic acid (IAA) (all plus $0.1 \%(\mathrm{v} / \mathrm{v})$ Tween-20). The leaves were collected $6 \mathrm{~h}$ after the spraying. Leaves sprayed with $0.1 \%$ Tween-20 were used as the control. 
RNA extraction, cDNA synthesis and real-time quantitative RT-PCR Total RNA from soybean was extracted using Trizol reagent (Invitrogen, USA) and then subjected to RNase-free DNase I (Promega, USA) digestion and purification. First strand cDNA was synthesized using AMV reverse transcriptase (BioFlux, Fluxion Biosciences, Inc., USA). Real-time quantitative PCR (qRT-PCR) was conducted using the CFX96 Real-Time PCR Detection System (Bio-Rad, USA). The amplification reaction was performed in a volume of $25 \mu \mathrm{L}$, which contains approximately $5 \mathrm{ng}$ template, $12.5 \mu \mathrm{L}$ SYBRGreen PCR Mastermix (Toyobo Japan) and $10 \mathrm{pmol}$ of each primer. The temperature profile was $98^{\circ} \mathrm{C}$ for $30 \mathrm{~s}$ followed by 40 cycles at $98^{\circ} \mathrm{C}$ for $2 \mathrm{~s}, 60^{\circ} \mathrm{C}$ for $5 \mathrm{~s}$ and a melt curve at $65^{\circ} \mathrm{C}$ for $5 \mathrm{~s}$. Cycle threshold values for each target gene were normalized according to those obtained in the corresponding reactions for the soybean elongation factor gene (accession no. EU790565.1). Relative expression was estimated using the $2^{-\Delta \Delta \mathrm{Ct}}$ method (Livak and Schmittgen, 2001). The primers used included: 5'-TGGAATGGTGGTGACTTTTGC-3' and 5'GGTCATCCTTTGAGTTTGAGGC-3' for $\mathrm{Gm} E F-1 a$; 5'TTCACCACCTTCACATCTT-3' and 5'-CGGTCAGTCTCGTAGTTC-3' for GmMlo4; 5'-AAGGATAGTTGGTGTGAGT$3^{\prime}$ and 5'-CAAGAGATACAGGAATGATAGC-3' for
GmMlo15; 5'-GAAGA GGAACACCGTAGG-3' and 5'TGAAGGACTGCTAAGAAGAATA-3' for GmMlo17; and 5'-ACACCACACCATTCTCAA-3' and 5'-GCACTCTCACTTCTTCCA-3' for GmMlo19.

\section{RESULTS}

Identification of Mlo genes in soybean To identify all the putative genes of the Mlo gene family in soybean, BLAST was used to search the soybean genome databases (http://www.phytozome.net/soybean.php, phytozome) using the HMM Profile of the MLO domain as a query. By using this approach, 20 putative soybean Mlo genes were identified. The EST database provided confirmative transcript information for all Mlo genes. These genes were designated sequentially from GmMlo1 to GmMlo20, according to their genome location (Table 1). The information of GmMlos, including accession numbers of the full-length cDNAs and proteins, and their physical locations on the chromosomes are listed in Table 1. Diverse exon-intron structures were identified by comparing the full-length cDNAs or predicted CDS with the genomic sequence of $\mathrm{GmMlo}$ genes in the soybean genome databases (Fig. 1). GmMlo exons ranged in number from 13 to 16 . There were $11 \mathrm{Gm} M l o$ genes containing a 5' or 3'

Table 1. Summary of GmMlo family members

\begin{tabular}{|c|c|c|c|c|c|c|c|c|c|}
\hline \multirow[b]{2}{*}{ Gene name } & \multirow[b]{2}{*}{ Locus } & \multirow[b]{2}{*}{ Peptide } & \multicolumn{3}{|c|}{ Gene structure } & \multicolumn{2}{|c|}{ Domain } & \multicolumn{2}{|c|}{ Character } \\
\hline & & & $\begin{array}{l}\text { Number } \\
\text { of Exon }\end{array}$ & $\begin{array}{l}\text { Number } \\
\text { of intron }\end{array}$ & UTR & Length & location & $\mathrm{TM}$ & CaMBD \\
\hline GmMlo1 & $01 g 36170.1$ & 597 & 14 & 13 & 5';3'UTR & 478 & $32-519$ & 7 & Yes \\
\hline GmMlo2 & 02g07110.1 & 589 & 16 & 15 & No & 507 & $7-513$ & 6 & Yes \\
\hline GmMlo3 & 03g22960.1 & 518 & 13 & 12 & 5';3'UTR & 497 & $6-502$ & 7 & Yes \\
\hline GmMlo4 & 04g00370.1 & 507 & 13 & 12 & 5';3'UTR & 489 & $6-494$ & 7 & Yes \\
\hline GmMlo5 & 04g01710.1 & 469 & 16 & 15 & No & 448 & $8-455$ & 5 & Yes \\
\hline GmMlo6 & 04g01730.1 & 545 & 14 & 13 & No & 490 & $7-496$ & 7 & Yes \\
\hline GmMlo7 & $06 \mathrm{~g} 00440.1$ & 498 & 15 & 14 & 5'UTR & 469 & $8-476$ & 7 & Yes \\
\hline GmMlo8 & 06g01820.1 & 542 & 14 & 13 & No & 483 & $7-489$ & 7 & Yes \\
\hline GmMlo9 & 08g20120.1 & 557 & 15 & 14 & No & 400 & $3-402$ & 6 & Yes \\
\hline GmMlo 10 & 10g35640.1 & 537 & 15 & 14 & 3'UTR & 436 & $1-436$ & 6 & Yes \\
\hline GmMlo11 & 11g09270.1 & 601 & 15 & 14 & 3'UTR & 422 & $1-422$ & 8 & Yes \\
\hline GmMlo12 & $11 \mathrm{~g} 15920.1$ & 599 & 15 & 14 & 3'UTR & 427 & $1-427$ & 7 & Yes \\
\hline GmMlo13 & 12g07530.1 & 578 & 15 & 14 & No & 403 & $1-402$ & 7 & Yes \\
\hline GmMlo14 & 12g29330.1 & 586 & 16 & 15 & 3'UTR & 410 & $1-411$ & 7 & Yes \\
\hline GmMlo15 & 13g35390.2 & 544 & 15 & 14 & 3'UTR & 477 & $11-487$ & 7 & Yes \\
\hline GmMlo16 & $15 \mathrm{~g} 13070.1$ & 509 & 15 & 14 & No & 396 & $3-398$ & 8 & No \\
\hline GmMlo17 & 16g21510.1 & 577 & 15 & 14 & 5';3'UTR & 412 & $1-412$ & 8 & Yes \\
\hline GmMlo18 & 16g26090.1 & 623 & 16 & 15 & No & 436 & $16-451$ & 7 & Yes \\
\hline GmMlo19 & $16 g 26100.1$ & 592 & 16 & 15 & 3'UTR & 402 & $12-413$ & 7 & Yes \\
\hline GmMlo20 & 20g31910.1 & 560 & 15 & 14 & No & 376 & $1-376$ & 6 & Yes \\
\hline
\end{tabular}

UTR, Untranslated region; TM, Transmembrane segments; CaMBD, CaM-binding domain. 


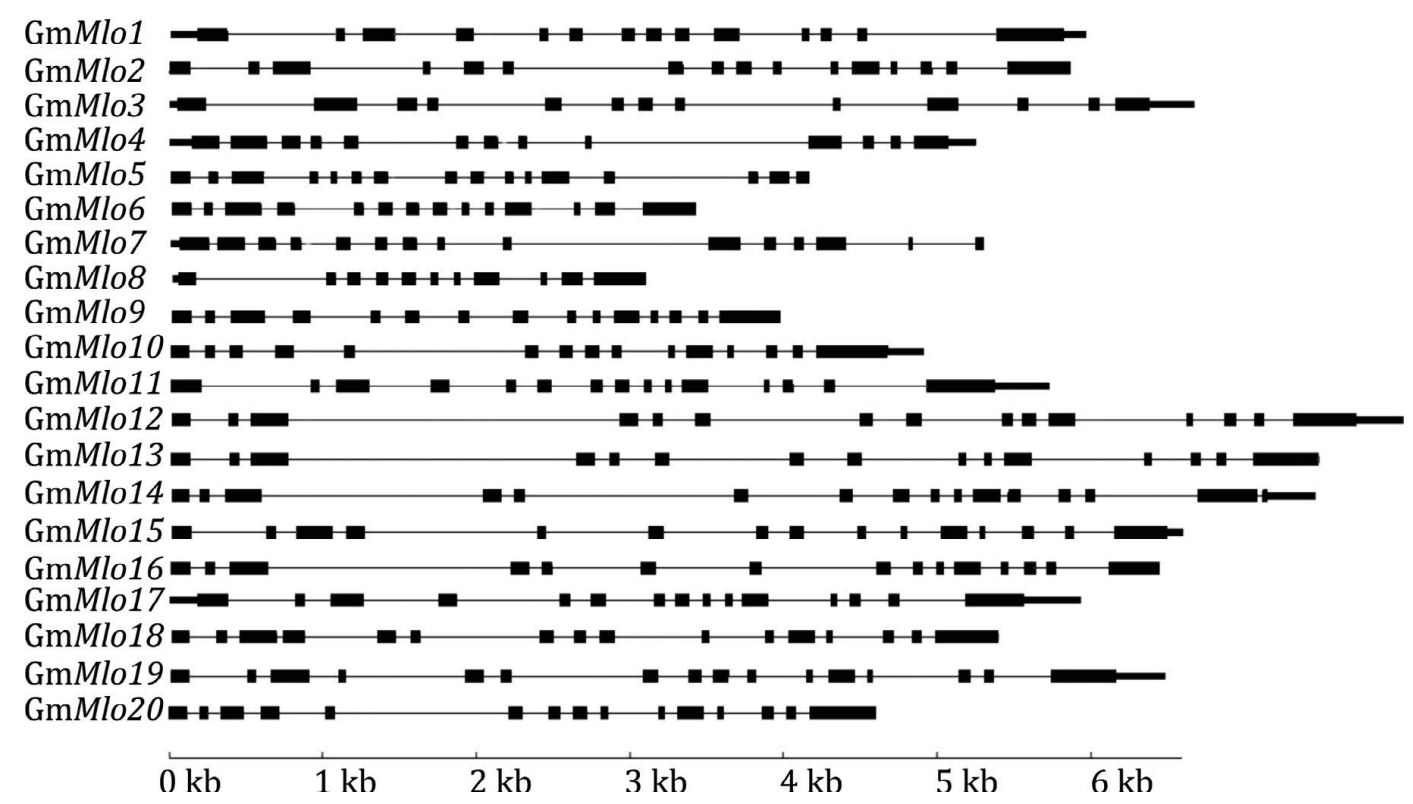

Fig. 1. Intron-exon organization of $20 \mathrm{GmMlo}$ genes. Exon(s) are shown in black and spaces between the black boxes correspond to introns.

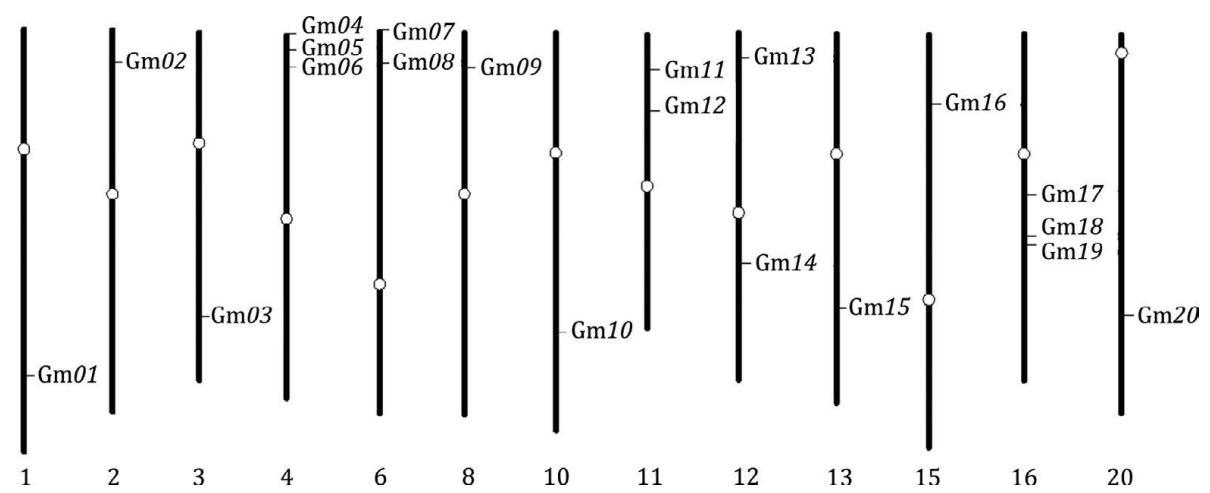

Fig. 2. Genomic distribution of GmMlo genes on soybean chromosomes. The bars represent the loci with the gene name on the right. A white circle on the chromosome indicates the approximate position of the centromeres. Chromosome numbers are indicated at the bottom of each chromosome.

un-translated region (UTR). The $20 \mathrm{GmMlo}$ genes were unevenly located on the 13 chromosomes (Fig. 2) and the number of genes ranged from one to three ranged per chromosomes. There was only one group, GmMlo18/19 that had a clustered distribution on the soybean chromosome, whereas the remainder had a scattered distribution. Meanwhile, nine GmMlo genes including GmMlo1, 11, 17, GmMlo2, 3, 18 and GmMlo12, 13, 14 were identified on segmental duplicated regions.

Sequence analysis of GmMLO proteins GmMLO proteins have a typically MLO domain. The length of the MLO proteins varied greatly, from a minimum of 469 (GmMLO5) to 623 amino acids (GmMLO18) (Table 1). The multiple alignments of the amino acids of GmMLOs showed considerable sequence diversity and the overall pairwise sequence identity was generally
$<60 \%$ (data not shown). However, more identity was found among the MLO domain of the GmMLO proteins (Fig. 3).

TM structures were predicted by using TMHMM2 and showed that the GmMLO proteins contained five to eight TM segments (Table 1); these putative TM segments were located on GmMLOs, based on sequence alignment (Fig. 3). An exact match for CaMBD, an important motif for the calcium response, was found on the C-terminal cytoplasmic tail of all the GmMLO proteins, with the exception of GmMLO16. Highly conserved tryptophan (Trp) residues and hydrophobic residues located at positions 1 , 8 and 14 were observed in the CaMBD-containing GmMLO proteins (Fig. 3).

Phylogenetic analysis of MLO proteins To gain insight into the potential function of different GmMLO pro- 
TM 1

TM2

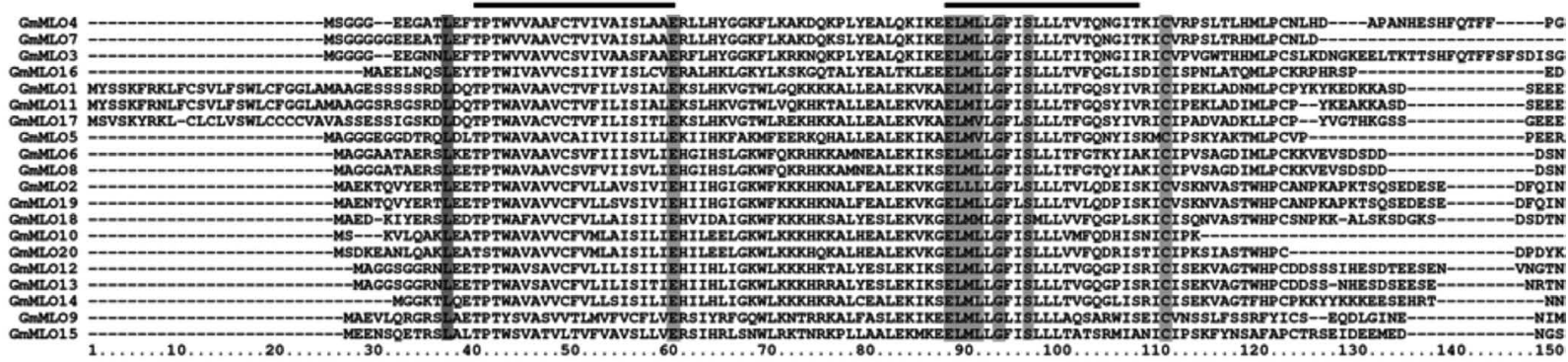

TM3

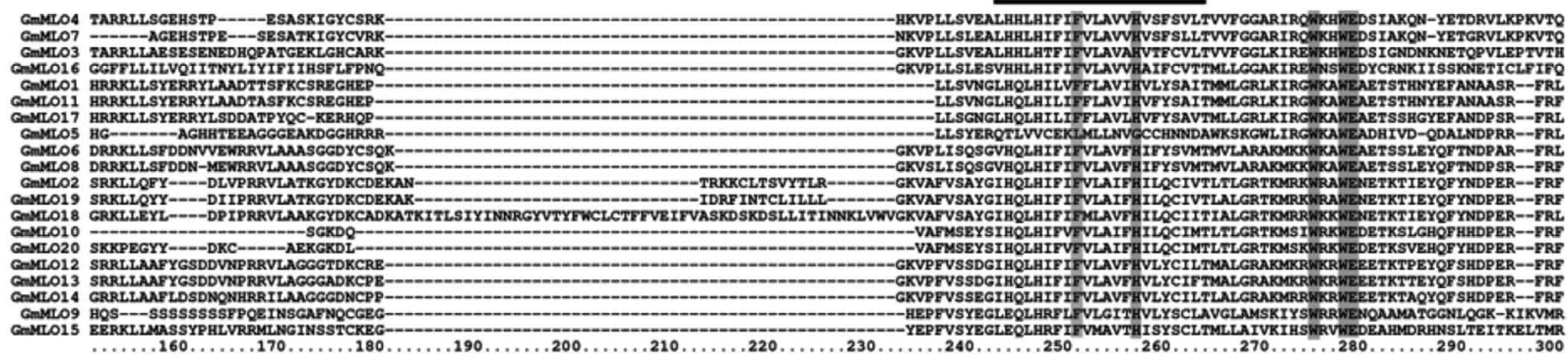

TM4

TM5

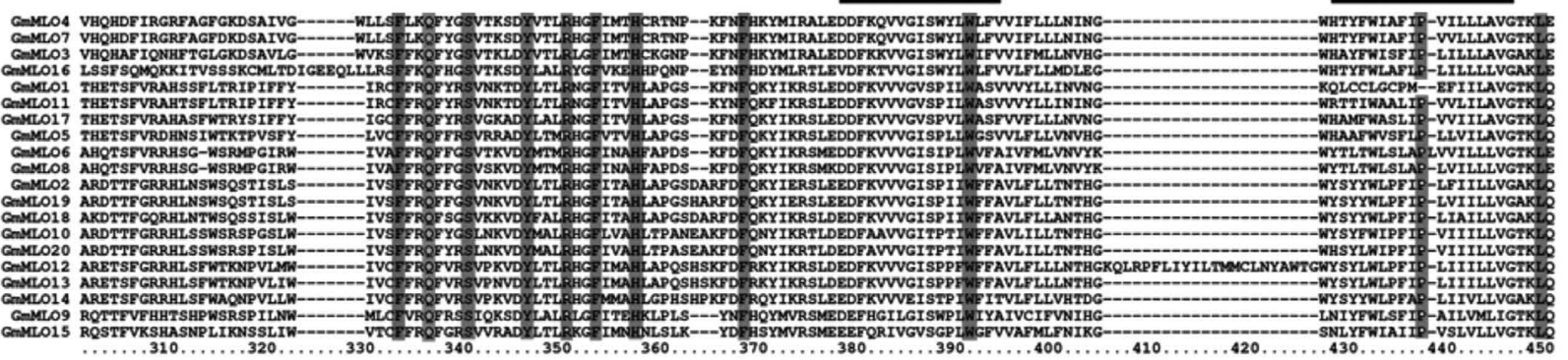

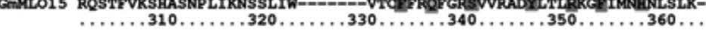

TM7

CaMBD
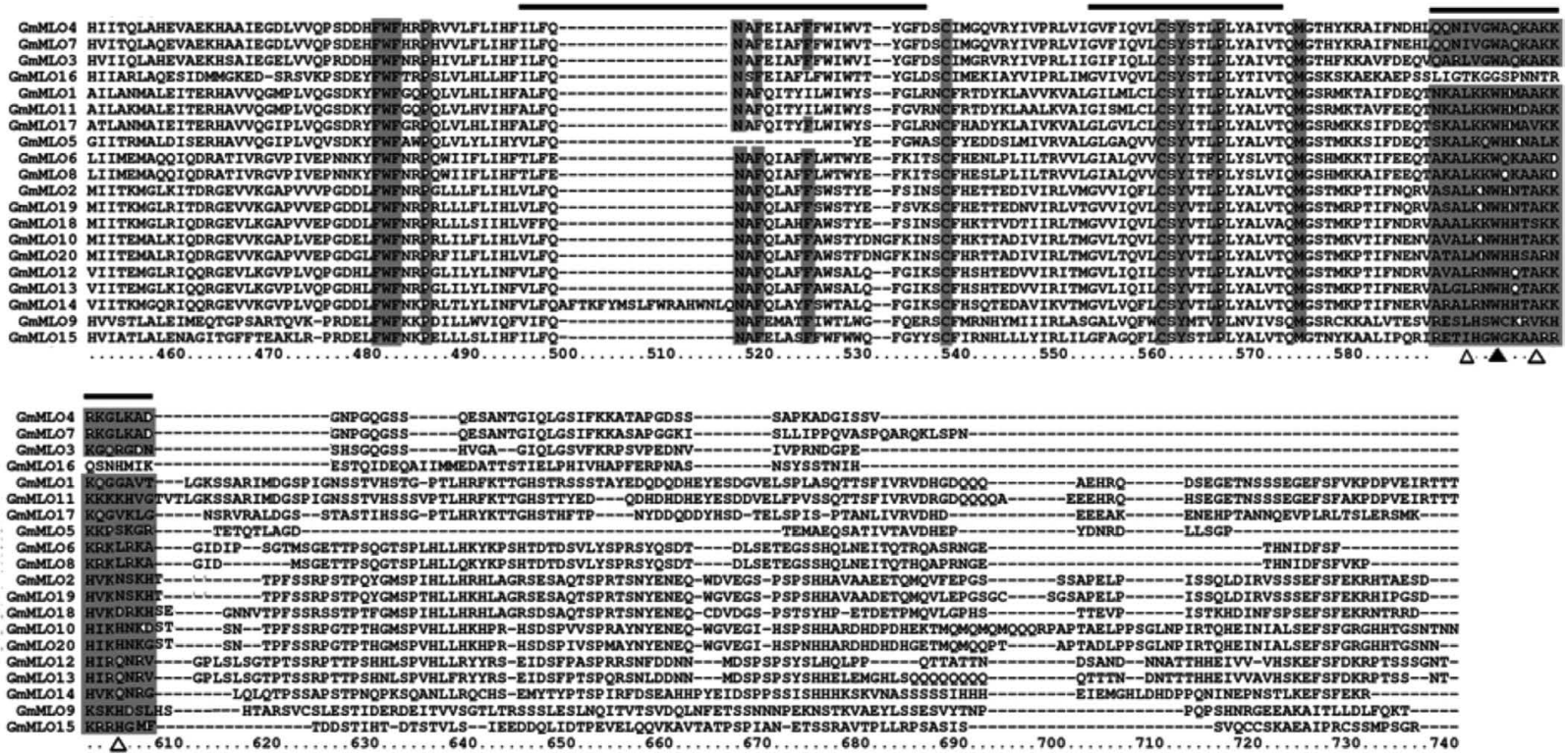

Fig. 3. Sequence alignments of GmMLO proteins. Dark shading indicates invariant residues. Black or white triangles indicate nonvariable Trp residues and highly conserved hydrophobic amino acids in the CaMDB domain.

teins, a phylogenetic tree was constructed to compare the relationship between MLOs from other plant species $(20$ in soybean; 15 in Arabidopsis; 9 in maize; 11 in rice (but not OsMLO10); 3 in barley; 6 in wheat; and 3 MLO proteins from other species) (Fig. 4). The results showed that the MLO family comprises four subfamilies and a 


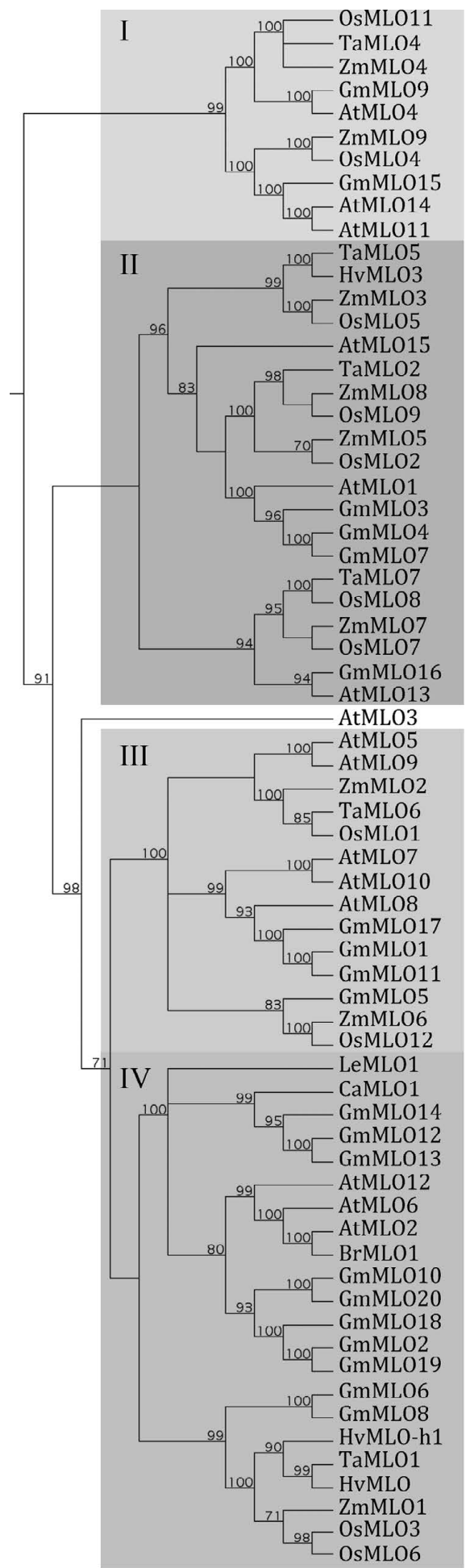

Fig. 4. Phylogenetic analysis of MLO proteins from soybean and other plant species. The Arabidopsis CRT1 protein (NP_001031199) was selected as the outgroup. The tree shows the four major phylogenetic subfamilies (numbered I to IV and marked with different alternating background to make subfamily identification easier) with high predictive value. At, Arabidopsis thaliana; $\mathrm{Br}$, Brassica rapa; $\mathrm{Ca}$, Capsicum annuum; $\mathrm{Hv}$, Hordeum vulgare; Le, Lycopersicon esculentum; Os, Oryza sativa; Pp, Physcomitrella patens; Ta, Triticum aestivum; Zm, Zea mays. single divergent lineage (AtMLO3). Subfamilies III and IV showed strong bootstrap support for a sister group relationship, and contained in total, $14 \mathrm{GmMlo}$ genes. Four GmMlo genes (GmMlo1, 5, 11, 17) belonged to subfamily III and ten GmMlo genes (GmMlo2, 6, 8, 10, 12, 13, $14,18,19,20$ ) belonged to subfamily IV. Other GmMlo9 and GmMlo15 belonged to subfamily I and four GmMlo genes $(\mathrm{GmMlo3}, 4,7,16)$ belonged to subfamily II.

Expression of GmMlos under abiotic stresses and phytohormone applications To find out whether $\mathrm{GmMlos}$ are response to abiotic stresses, the expression of $\mathrm{Gm} M l o$ genes was examined in plants subjected to abiotic treatments (heat, cold, drought and salinity). One $\mathrm{Gm} M l o$ gene was selected from each subfamily. The expression of four GmMlo genes (GmMlo4, 15, 17, 19) was analyzed (Fig. 5). After heat treatment, the transcription levels of four genes were downregulated (Fig. 5A). Under cold conditions, the expression of GmMlo15 and GmMlo17 was upregulated, whereas that of GmMlo19 was downregulated; however, this treatment had no effect on GmMlo4 (Fig. 5B). As shown in Fig. 5C, GmMlo4 was upregulated under drought conditions, whereas the other three genes were downregulated (Fig. 5C). GmMlo15 and GmMlo17 were downregulated under the salinity treatment, although, this treatment had no effect on either GmMlo4 or GmMlo19 (Fig. 5D).

Given that the abiotic stress response involves phytohormone signaling pathways, the expression of GmMlo genes was examined in response to exogenous application of ABA, 6-BA, IAA and $\mathrm{GA}_{3}$. As shown in Fig. 5 (E and F), four genes were suppressed in leaves were sprayed with either $10 \mathrm{mM}$ ABA or $1 \mathrm{mM} \mathrm{6-BA.} \mathrm{GmMlo4} \mathrm{and}$ GmMlo15, 17, and 19 expressions was also up- or downregulated in leaves sprayed with $1 \mathrm{mM}$ IAA (Fig. 5G). GmMlo15 showed no response in leaves sprayed with $10 \mathrm{mM} \mathrm{GA}_{3}$, whereas GmMlo4 and GmMlo17 expression was induced, but that of GmMlo19 was downregulated (Fig. 5H). The strong response of $\mathrm{GmMlos}$ following abiotic stress and phytohormone treatments suggests that they have various roles in the responses of environmental stimuli.

cis-response element analysis of the promoters of GmMlo genes The promoter region of the GmMlo genes (2,0-kb upstream of the transcriptional start site) was analyzed using the Plant CARE database to identify putative stress-responsive cis-elements. Sequence analysis identified a total of nine types of stress-related cisregulatory elements among GmMlo genes (Table 2). The defense-responsive cis-regulatory elements contained a putative TC-rich repeats element. Abiotic responsive cis-elements, such as the heat-stress element (HSE), were identified in 12 and 16 promoters of $\mathrm{GmMlo}$ genes. The drought responsiveness element, which contains MYB 
A

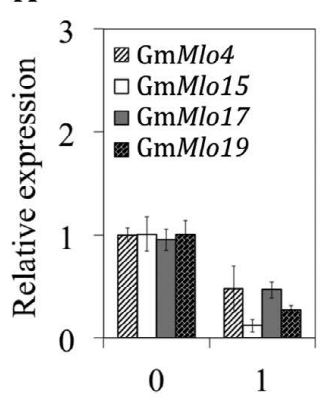

E

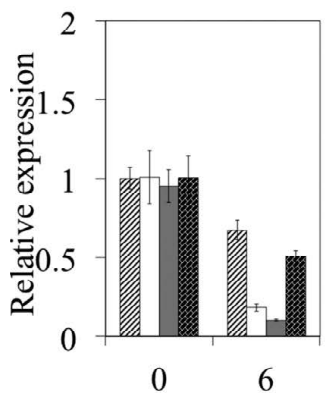

B

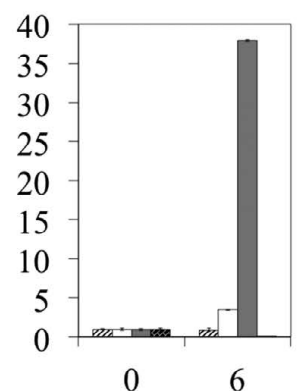

F

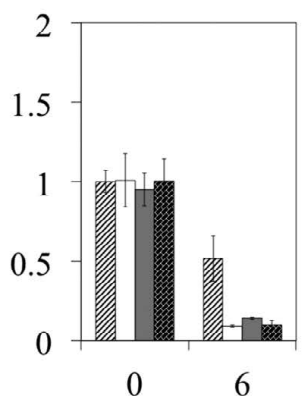

C

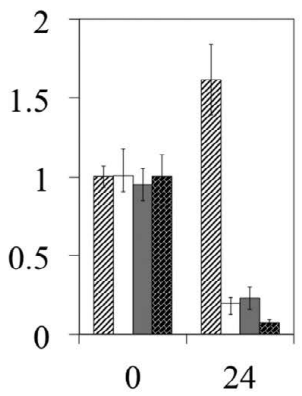

24

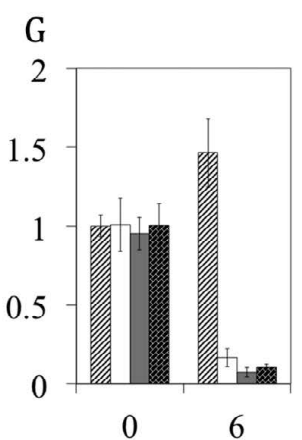

D
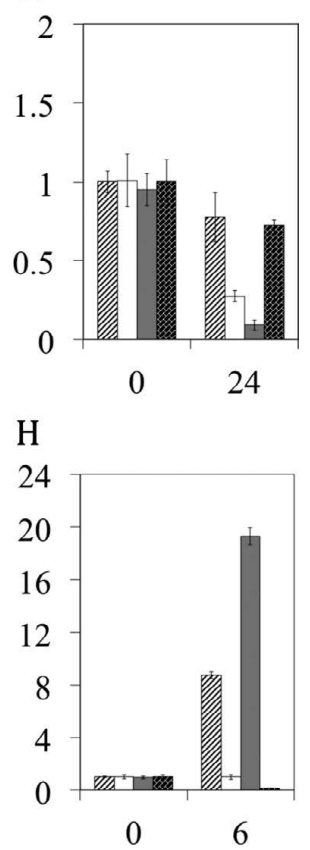

Fig. 5. Expression of GmMlo genes in response to abiotic treatments and phytohormone applications. (A) heat treatment, (B) cold treatment, (C) drought treatment, (D) salinity treatment, (E) ABA treatment, (F) 6-BA treatment, (G) IAA treatment and (H) GA3 treatment. '0' represents a simulated treatment. The horizontal axis represents the time (h) after either abiotic or phytohormone treatments. Error bars represent the standard deviation from three replicated experiments.

Table 2. Putative regulatory cis-element sequences in the promoter of GmMlo genes

\begin{tabular}{|c|c|c|c|c|c|c|}
\hline \multirow[b]{2}{*}{$\begin{array}{c}\text { Element } \\
\text { name }\end{array}$} & Defense & Heat & Drought & Auxin & $\mathrm{ABA}$ & Gibberellin \\
\hline & $\begin{array}{l}\text { TC-rich } \\
\text { repeats }\end{array}$ & HSE & $\begin{array}{c}\text { MYB } \\
\text { binding site/ } \\
\text { DRE }\end{array}$ & AuxRE & ABRE & $\begin{array}{c}\text { GARE-motif/ } \\
\text { TATC-box/ } \\
\text { P-box }\end{array}$ \\
\hline GmMlo1 & + & + & + & & + & \\
\hline GmMlo2 & + & + & + & & & + \\
\hline GmMlo3 & & + & + & + & & \\
\hline GmMlo4 & + & & & & + & + \\
\hline GmMlo5 & + & & + & & + & \\
\hline GmMlo6 & + & + & + & & & + \\
\hline GmMlo7 & + & + & & & & \\
\hline GmMlo8 & & + & + & & + & \\
\hline GmMlo9 & & & + & & + & + \\
\hline GmMlo 10 & + & + & & & + & \\
\hline GmMlo 11 & & + & + & & + & \\
\hline GmMlo 12 & & + & & & & \\
\hline GmMlo13 & & + & & & + & \\
\hline GmMlo 14 & + & + & + & + & + & \\
\hline GmMlo15 & + & & + & & & + \\
\hline GmMlo 16 & + & + & + & & & + \\
\hline GmMlo17 & + & + & + & & + & + \\
\hline GmMlo18 & + & + & + & & + & \\
\hline GmMlo19 & & + & + & & + & \\
\hline GmMlo20 & & + & & & & \\
\hline
\end{tabular}

binding site and a dehydration responsive element (DRE), was identified in 14 promoters of GmMlo genes; The ABA responsive element (ABRE), gibberellin-responsive element (containing a GARE-motif, TATC-box and P-box) and auxin-responsive element (AuxRE) were also identified in 12, 7 and 2 promoters of $\mathrm{GmMlo}$ genes, respectively (Table 2).

Major stress-responsive genes contain putative corresponding stress-responsive cis-elements in their promoters. For example, the $\mathrm{GA}_{3}$-responsive element was identified in the promoter region of $\mathrm{GmMlo4}$ and GmMlo17, which were induced by GA3 treatment, GmMlo19 were also induced in response to $\mathrm{GA}_{3}$ treatments, and the corresponding elements was identified in its promoters. However, the putative cis-elements detected in GmMlo genes were not closely related with the stress responsiveness. GARE was identified in the promoter of GmMlo15, but the gene did not show a change in response to GA3 treatment. ABA-responsive elements were identified in the promoter of GmMlo19, despite the fact that the gene was downregulated after in leaves treated with ABA.

\section{DISCUSSION}

Mlo is a multi-membered gene family. So far, 15,12 , 9 and 7 Mlo genes have been identified in Arabidopsis thaliana, rice, maize and wheat, respectively. Mean- 
while, a few Mlo genes have been identified and isolated from barley, tomato Lycopersicon, pepper Capsicum and cabbage Brassica, respectively (Devoto et al., 2003; Miklis et al., 2007; Liu and Zhu, 2008; Konishi et al., 2010). In the current study, 20 MLO domain-containing sequences were identified in soybean, based on database searches and analysis (Table 1). The GmMlos were similar in character to other Mlo gene members in Arabidopsis, barley and wheat; for example, in terms of their TM and CaMBD structures. The seven TM structures are thought to be a key characteristic of the Mlo gene family (Devoto et al., 2003; Elliott et al., 2005; Feechan et al., 2008). Seven TM segments were also located on GmMLO members based on sequence alignment. However, as predicted by TMHMM2, the GmMLO proteins could contain five to eight TM segments (Table 1). The various TM structures might be the result of structural and allosteric complexity that is too difficult to be predicted by computational methods (Devoto et al., 1999; Kenakin, 2009). Therefore, the occurrence of seven TM segments is also thought to be a main characteristic among GmMLO proteins. The CaMBD motif located on the C-terminus in MLO family members has been identified as an important $\mathrm{Ca}^{2+}$ signal response motif that modulates defence reactions against powdery mildew (Kim et al., 2002a, 2002b). Among the 20 predicted GmMlo genes, 19 genes have the conserved CaMBD motif. GmMlo6 did not show any detectable CaMBD motif, but it may be a special Mlo gene in soybean genome, also, it may be a pseudogene.

GmMlo genes were distributed on 13 soybean chromosomes, with a mainly scattered distribution expect one group existed a clustered distribution. Gene families could arise through tandem amplification or segmental duplication of chromosomal regions. Tandem amplification results in a clustered distribution, whereas segmental duplication results in a scattered distribution (Schauser et al., 2005). Therefore, it is suggested that segmental duplication is the main amplification method that occurs in GmMlo family members; in the current study, three groups of $\mathrm{GmMlo}$ genes were identified from one segmental duplication event. The result is similar to AtMlos and OsMlos family members, which also mainly result from segmental duplication (Devoto et al., 2003; Liu and Zhu, 2008). Meanwhile, the existence of multiple $M l o$ gene copies in soybean is not surprising, soybean is a paleopolyploid, also, paleopolyploid must have been the factor of gene duplication. Moreover, the relationship of segmental duplication with clustering in the phylogenetic tree was appeared, It seems that members derived from segmental chromosome duplication such as GmMlo1, 11, 17 are clustered together in the same subfamily.

The response of Mlo family members to $\mathrm{Ca}^{2+}$ signals to modulated plant defence reactions against powdery mil- dew and to regulated cell death has been identified (Kim et al., 2002a, 2002b; Bai et al., 2008). Increasing evidences suggests that $M l o$ genes have additional functions. Their transcription can occur in response to wounding, paraquat, and auxins and phytohormone stress (Piffanelli et al., 2002; Feechan et al., 2008). In the current study, four $\mathrm{GmMlos}$ were responsive to various abiotic stresses and phytohormone treatments. Multi-type abiotic- and phytohormone-responsive elements predicted in the promoters of the GmMlo genes were also take as evidence of the various stress-responsive functions of GmMlos. The ABA-, gibberellin- and auxin-responsive elements, in particular, were identified in parts of promoters of $\mathrm{GmMlo}$ genes. ABA is an important phytohormone that can convert the initial stress signal, such as drought or high salinity into a cellular response (Fujita et al., 2005; Nakashima et al., 2009). Auxins and gibberellins are also important signal molecules in environmental signal transduction (Sun, 2000; Berleth et al., 2004). In addition, crosstalk between phytohormones and $\mathrm{Ca}^{2+}$ signals in response to various environmental stresses has been reported (Van der Meulen et al., 1996; Gao et al., 2002; Yang and Poovaiah, 2003; Navarro-Avino and Bennet, 2005; Qudeimat et al., 2008; Siegel et al., 2009; Kang et al., 2010; Zou et al., 2010). However, this evidence comes mainly from Arabidopsis. To date, many questions remain relating to the mechanisms of MLO proteins. Crosstalk between abiotic stresses and phytohormone signaling pathways increases the complexity of MLO function. Isolation and characterization of Mlo genes on a genome scale could pave the way for functional investigations. The results from the current study provide obvious clues for further elucidating the functions of members of the Mlo genes family in response to environmental stimuli.

This work was partially supported by Research Fund for the Doctoral Program of Higher Education of China (No.20090097120023) and Science and Technology Foundation of Guizhou province (No. [2010] 2089).

\section{REFERENCES}

Bai, Y., Pavan, S., Zheng, Z., Zappel, N. F., Reinstädler, A., Lotti, C., De Giovanni, C., Ricciardi, L., Lindhout, P., Visser, R., et al. (2008) Naturally occurring broad-spectrum powdery mildew resistance in a Central American tomato accession is caused by loss of Mlo function. Mol. Plant-Microbe. Interact. 21, 30-39.

Berleth, T., Krogan, N. T., and Scarpella, E. (2004) Auxin signals-turning genes on and turning cells around. Curr. Opin. Plant Biol. 7, 553-563.

Büschges, R., Hollricher, K., Panstruga, R., Simons, G., Wolter, M., Frijters, A., van Daelen, R., van der Lee, T., Diergaarde, P., Groenendijk, J., et al. (1997) The barley Mlo gene: A novel control element of plant pathogen resistance. Cell 88, 695-705.

Consonni, C., Humphry, M. E., Hartmann, H. A., Livaja, M., 
Durner, J., Westphal, L., Vogel, J., Lipka, V., Kemmerling, B., Schulze-Lefert, P., et al. (2006) Conserved requirement for a plant host cell protein in powdery mildew pathogenesis. Nat. Genet. 38, 716-720.

Consonni, C., Bednarek, P., Humphry, M., Francocci, F., Ferrari, S., Harzen, A., Ver Loren van Themaat, E., and Panstruga, R. (2010) Tryptophan-derived metabolites are required for antifungal defense in the Arabidopsis mlo2 mutant. Plant Physiol. 152, 1544-1561.

Devoto, A., Piffanelli, P., Nilsson, I., Wallin, E., Panstruga, R., von Heijne, G., and Schulze-Lefert, P. (1999) Topology, subcellular localization, and sequence diversity of the Mlo family in plants. J. Biol. Chem. 274, 34993-35004.

Devoto, A., Hartmann, H. A., Piffanelli, P., Elliott, C., Simmons, C., Taramino, G., Goh, C. S., Cohen, F. E., Emerson, B. C., Schulze-Lefert, P., et al. (2003) Molecular phylogeny and evolution of the plant-specific seven-transmembrane MLO family. J. Mol. Evol. 56, 77-88.

Du, L., Ali, G. S., Simons, K. A., Hou, J., Yang, T., Reddy, A. S., and Poovaiah, B. W. (2009) $\mathrm{Ca}\left({ }^{2+}\right) /$ calmodulin regulates salicylic-acid-mediated plant immunity. Nature 457, 11541158.

Elliott, C., Müller, J., Miklis, M., Bhat, R. A., Schulze-Lefert, P., and Panstruga, R. (2005) Conserved extracellular cysteine residues and cytoplasmic loop-loop interplay are required for functionality of the heptahelical MLO protein. J. Biochem. 385, 243-254.

Feechan, A., Jermakow, A. M., Torregrosa, L., Panstruga, R., and Dry, I. B. (2008) Identification of grapevine $M L O$ gene candidates involved in susceptibility to powdery mildew. Funct. Plant Biol. 35, 1255-1266.

Finn, R. D., Mistry, J., Schuster-Böckler, B., Griffiths-Jones, S., Hollich, V., Lassmann, T., Moxon, S., Marshall, M., Khanna, A., Durbin, R., et al. (2006) Pfam: clans, web tools and services. Nucleic Acids Res. 34 (Suppl. 1), D247-D251.

Fujita, Y., Fujita, M., Satoh, R., Maruyama, K., Parvez, M. M., Seki, M., Hiratsu, K., Ohme-Takagi, M., Shinozaki, K., and Yamaguchi-Shinozaki, K. (2005) AREB1 is a transcription activator of novel ABRE-dependent ABA-signaling that enhances drought stress tolerance in Arabidopsis. Plant Cell 17, 3470-3488.

Gao, Y. P., Bonham-Smith, P. C., and Gusta, L. V. (2002) The role of peroxiredox in antioxidant and calmodulinin ABAprimed seeds of Brassica napus exposed to abiotic stresses during germination. J. Plant Physiol. 159, 951-958.

Hrabak, E. M., Chan, C. W. M., Gribskov, M., Harper, J. F., Choi, J. H., Halford, N., Kudla, J., Luan, S., Nimmo, H. G., Sussman, M. R., et al. (2003) The Arabidopsis CDPK-SnRK superfamily of protein kinases. Plant Physiol. 132, 666680.

Jørgensen, I. H. (1992) Discovery, characterization and exploitation of Mlo powdery mildew resistance in barley. Euphytica 63, 141-152.

Kang, S. M., Kim, J. T., Hamayun, M., Hwang, I. C., Khan, A. L., Kim, Y. H., Lee, J. H., and Lee, I. J. (2010) Influence of prohexadione-calcium on growth and gibberellins content of Chinese cabbage grown in alpine region of South Korea. Scientia Horticulturae 125, 88-92.

Kenakin, T. P. (2009) '7 TM Receptor Allostery: Putting numbers to shape shifting proteins. Trends Pharmacol. Sci. 30, 460-469.

Kim, M. C., Lee, S. H., Kim, J. K., Chun, H. J., Choi, M. S., Chung, W. S., Moon, B. C., Kang, C. H., Park, C. Y., Yoo, J. H., et al. (2002a) Mlo, a modulator of plant defense and cell death, is a novel calmodulin-binding protein. J. Biol.
Chem. 277, 19304-19314.

Kim, M. C., Panstruga, R., Elliott, C., Müller, J., Devoto, A., Yoon, H. W., Park, H. C., Cho, M. J., and Schulze-Lefert, P. (2002b) Calmodulin interacts with MLO protein to regulate defence against mildew in barley. Nature 416, 447-450.

Konishi, S., Sasakuma, T., and Sasanuma, T. (2010) Identification of novel Mlo family members in wheat and their genetic characterization. Gene Genet. Syst. 85, 167-175.

Krogh, A., Larsson, B., von Heijine, G., and Sonnhammer, E. L. (2001) Predicting transmembrane protein topology with hidden Markov model: application to complete genomes. J. Mol. Biol. 305, 567-580.

Lescot. M., Déhais, P., Thijs, G., Marchal, K., Moreau, Y., Van de Peer, Y., Rouzé, P., and Rombauts, S. (2002) PlantCARE, a database of plant cis-acting regulatory elements and a portal to tools for in silico analysis of promoter sequences. Nucleic Acid Res. 30, 325-327.

Letunic, I., Copley, R. R., Schmidt, S., Ciccarelli, F. D., Doerks, T., Schultz, J., Ponting, C. P., and Bork, P. (2004) SMART4.0: towards genomic data integration. Nucleic Acids Res. 32, 142-144.

Liu, Q., and Zhu, H. (2008) Molecular evolution of the $M L O$ gene family in Oryza sativa and their functional divergence. Gene 409, 1-10.

Livak, K. J., and Schmittgen, T. D. (2001) Analysis of relative gene expression data using real-time quantitative PCR and the $2^{-\Delta \Delta \mathrm{CT}}$ method. Methods 25, 402-408.

Miklis, M., Consonni, C., Bhat, R. A., Lipka, V., Schulze-Lefert, P., and Panstruga R. (2007) Barley MLO modulates actindependent and actin-independent antifungal defense pathways at the cell periphery. Plant Physiol. 144, 1132-1143.

Nakashima, K., Ito, Y., and Yamaguchi-Shinozaki, K. (2009) Transcriptional regulatory networks in response to abiotic stresses in Arabidopsis and grasses. Plant Physiol. 149, 88-95.

Navarro-Aviñó, J. P., and Bennett, A. B. (2005) Role of a $\mathrm{Ca}^{2+}$ ATPase induced by ABA and IAA in the generation of specific $\mathrm{Ca}^{2+}$ signals. Biochem. Biophys. Res. Commun. 329, $406-415$.

Piffanelli, P., Zhou, F., Casais, C., Orme, J., Jarosch, B., Schaffrath, U., Collins, N. C., Panstruga, R., and SchulzeLefert, P. (2002) The barley $M L O$ modulator of defense and cell death is responsive to biotic and abiotic stress stimuli. Plant Physiol. 129, 1076-1085.

Piffanelli, P., Ramsay, L., Waugh, R., Benabdelmouna, A. D’Hont, A., Hollricher, K., Jørgensen, J. H., Schulze-Lefert, P., and Panstruga, R. (2004) A barley cultivation-associated polymorphism conveys resistance to powdery mildew. Nature 430, 887-891.

Qudeimat, E., Faltusz, A. M. C., Wheeler, G., Lang, D., Holtorf, H., Brownlee, C., Reski, R., and Frank, W. (2008) A PIIBtype $\mathrm{Ca}^{2+}$-ATPase is essential for stress adaptation in Physcomitrella patens. Proc. Acad Natl. Sci. USA. 105, 19555-19560.

Reddy, A. S. (2001) Calcium: silver bullet in signaling. Plant Sci. 160, 381-404.

Rudd, J. J., and Franklin-Tong, V. E. (2001) Unravelling response-specificity in $\mathrm{Ca}^{2+}$ signalling pathways in plant cells. New Phytol. 151, 7-33.

Sanders, D., Pellouxa, J., Brownlee, C., and Harper, J. F. (2002) Calcium at the crossroads of signaling. Plant Cell 14, 401417.

Schauser, L., Wieloch, W., and Stougaard, J. (2005) Evolution of NIN-Like proteins in Arabidopsis, rice, and Lotus japonicus. J. Mol. Evol. 60, 229-237. 
Siegel, R. S., Xue, S., Murata, Y., Yang, Y., Nishimura, N., Wang, A., and Schroeder, J. I. (2009) Calcium elevation-dependent and attenuated resting calcium-dependent abscisic acid induction of stomatal closure and abscisic acid-induced enhancement of calcium sensitivities of S-type anion and inward-rectifying $\mathrm{K}$ channels in Arabidopsis guard cells. Plant J. 59, 207-220.

Stein, M., and Somerville, S. C. (2002) MLO, a novel modulator of plant defenses and cell death, binds calmodulin. Trends Plant Sci. 7, 379-380.

Sun, T. P. (2000) Gibberellin signal transduction. Curr. Opini. Plant Biol. 3, 374-380.

Van der Meulen, R. M., Visser, K., and Wang, M. (1996) Effects of modulation of calcium levels and calcium fluxes on ABAinduced gene expression in barley aleurone. Plant Sci. 117, $75-82$.

Wang, L., Tsuda, K., Sato, M., Cohen, J. D, Katagiri, F., and Glazebrook, J. (2009) Arabidopsis CaM binding protein
CBP60g contributes to MAMP-induced SA accumulation and is involved in disease resistance against Pseudomonas syringae. PLoS Pathog. 5, e1000301.

Wolter, M., Hollricher, K., Salamini, F., and Schulze-Lefert, P. (1993) The mlo resistance alleles to powdery mildew infection in barley trigger a developmentally controlled defense mimic phenotype. Mol. Gen. Genet. 239, 122-128.

Yang, T., and Poovaiah, B. W. (2003) Calcium/calmodulin-mediated signal network in plants. Trends Plant Sci. 8, 505512.

Zielinski, R. E. (1998) Calmodulin and calmodulin-binding proteins in plants. Ann. Rev. Plant Physiol. Plant Mol. Biol. 49, 697-725.

Zou, J. J., Wei, F. J., Wang, C., Wu, J. J., Ratnasekera, D., Liu, W. X., and Wu, W. H. (2010) Arabidopsis calcium-dependent protein kinase CPK10 functions in abscisic acid- and $\mathrm{Ca}^{2+}$ mediated stomatal regulation in response to drought stress. Plant Physiol. 154, 1232-1243. 